\title{
Responsabilidad social ambiental de las universidades colombianas
}

\author{
Environmental social responsibility \\ of colombian universities
}

\author{
Alberto García Jerez ${ }^{1}$ y Lucas Fernando Quintana Fuentes ${ }^{2}$ \\ 'Biólogo, Especialista en Química Ambiental y Candidato a Magister en Desarrollo Sostenible y \\ Medio Ambiente de la Universidad de Manizales. ${ }^{2}$ ngeniero de Alimentos, Especialista en Dirección \\ de Empresas y Magister en Calidad y Productividad. \\ 1,2 Escuela de Ciencias Básicas, Tecnología e Ingeniería (ECBTI). Universidad Nacional \\ Abierta y a Distancia UNAD. Bucaramanga. Colombia. \\ 1alberto.garcia@unad.edu.co, ${ }^{2}$ lucas.quintana@unad.edu.co
}

\begin{abstract}
Resumen
La responsabilidad social ambiental va más allá de los diferentes tratados internacionales, de la normatividad vigente o de la rigurosidad de las autoridades ambientales. Implica no solo el cumplimiento de la norma, sino también el esfuerzo extra, que tiene por objeto la producción limpia, disminuir los impactos al medio ambiente, a los ecosistemas y, por ende, a las comunidades humanas. Todas las actividades humanas que contaminan y destruyen la vida del planeta, pueden ser cambiadas o corregidas implementado nuevas tecnologías o simplemente con cambios de actitud. Dentro de todos los actores responsables en la búsqueda de soluciones a esta problemática, la universidad cumple con su desarrollo social ambiental al permear las comunidades, las industrias y el propio estado con programas académicos acordes con las necesidades locales o regionales de una población.
\end{abstract}

Palabras clave: responsabilidad social, ambiente, academia, universidad

\begin{abstract}
Environmental social responsibility goes beyond the various international treaties, current regulations, and stringency of the environmental authorities. It implies not only compliance with the law, but also the extra effort which aims to clean production, reduce environmental impacts on ecosystems and human communities they support. All human activities that pollute and destroy the planet's life can be changed or corrected by implementing new technologies or simply by changing attitudes. Amongst the parties responsible for finding solutions to this problem, the university fulfills its social environmental development in order to permeate communities, industries and the state itself according to local or regional necessities of a population.
\end{abstract}

Key words: social responsibility, environment, academy, university 


\section{Introducción}

La responsabilidad social ambiental puede entenderse como el cumplimiento de una serie de normas ambientales, pero en realidad transversa la dimisión ética y son acciones autónomas que comprometen y propenden por condiciones ambientales sanas. La responsabilidad social ambiental es concordante con el desarrollo sostenible, de una industria, país o comunidad; regido por el progreso en la economía, ecosistemas saludables y el bienestar integral. Estas acciones dependen de iniciativas de las comunidades, de la industria, del estado y de la academia, que debe concebir una legislación ambiental acorde con las realidades que afectan el país.

La legislación ambiental en Colombia se adopta a partir de la Convención de Estocolmo en 1972, se estableció el código de recursos naturales renovables y de protección al medio ambiente (Decreto Ley 2811 de 1974). La normatividad ambiental Colombiana se torna dinámica a partir de la Constitución Política de 1991 (Capítulo III "De los derechos colectivos y del Ambiente", los cuales establecen el marco general a partir del cual toda la política ambiental Colombiana debe desarrollarse).

Así, la responsabilidad social ambiental va más allá de los diferentes tratados internacionales, de la normatividad vigente o de la rigurosidad de las autoridades ambientales. Implica no solo el cumplimiento de la norma, sino también un esfuerzo extra, que tiene por objeto la producción limpia, disminuir los impactos al medio ambiente, a los ecosistemas y, por ende, a las comunidades humanas. Todas las actividades humanas que contaminan y destruyen la vida del planeta, pueden ser cambiadas o corregidas implementado nuevas tecnologías o simplemente con cambios de actitud. Dentro de todos los actores responsables en la búsqueda de soluciones a esta problemática, la universidad cumple con su desarrollo social ambiental al permear las comunidades, las industrias y el propio estado con programas académicos acordes con las necesidades locales o regionales de una población.
El desarrollo de la conciencia social ambiental, reside en la estrategia educativa que busca dar soluciones al inminente deterioro de los ecosistemas y al agotamiento de los recursos naturales explotados por décadas, lo que ha causado daños irreversibles a corto y mediano plazo en el paisaje, la salud humana y en la vida silvestre.

Dentro de este contexto de la educación ambiental, hasta hace cinco décadas, la ausencia de una conciencia global sobre los cambios realizados al ambiente, produjeron destrucción y muerte de diversas especies. Las primeras investigaciones sobre el deterioro ambiental se plasmaron en el libro Primavera silenciosa en 1962, cuya autora Rachel Louise Carson, llamaba la atención sobre sucesos que afectan las especies locales de aves y reptiles. Por primera vez, muestran, sustancias con el Dicloro Difenil Tricloroetano (DDT), responsables de grandes alteraciones en los ecosistemas y la muerte de especímenes de aves, reptiles, mamíferos y numerosas personas que interactuaban directamente con la sustancia, así como la sospecha de numerosas muertes asociadas a diferentes tipos de cáncer.

\section{Desarrollo}

Primavera Silenciosa fue la primera declaración pública, sobre la acción funesta de sustancias químicas, usadas para control de malezas, hongos, roedores y reptiles. Después de numerosos estudios y tras un par de décadas, se comprobó la persistencia de estas sustancias en los suelos, aguas, tejidos animales y vegetales.

En la actualidad la problemática ambiental se ha complejizado, diferentes fuentes de contaminación, consecuencia en gran parte del acelerado incremento de la población de humanos durante los últimos 50 años, da como resultante la sobre explotación del planeta tierra. La contaminación de las fuentes hídricas es tal vez la más visible, pero existen otros orígenes de contaminación como son la atmosférica, 
electromagnética, radioactiva, sonora, visual, química, biológica, transgénicas entre otras y todas estas muestran cambios globales ambientales.

La educación ambiental en América Latina surge de las comunidades campesinas e indígenas que plantean soluciones a problemáticas ambientales, teniendo como premisa el respeto por la tierra que sustenta la vida y permite que las comunidades se desarrollen en armonía con los ecosistemas y uso racional de los recursos naturales, contrario al ecologismo que esboza la protección de especies o ecosistemas sin integrar las soluciones ambientales con las comunidades.

Los principales investigadores ambientales surgen de las facultades de universidades que cuentan con programas académicos como zootecnia, alimentos, agroindustriales y civiles entre otras e institutos estatales relacionados con actividades del agro, las cuales tangencialmente realizan aportes incipientes a una problemática ambiental que ha dejado cicatrices en la tierra y alteración en los ecosistemas, pasando desapercibidos por la falta estudios previos.

Los gobiernos de Latinoamérica han preferido los conocimientos y tecnologías foráneas las cuales al ser aplicadas al contexto de los países ecuatoriales no han sido eficientes. La introducción de especies vegetales, animales, transgénicos y los programas implementados por gobiernos de países ricos y organismos internacionales de la banca han experimentado y explotado los recursos naturales que han cambiado el paisaje, han atropellado las comunidades hasta perderse en unas cuantas generaciones, culturas ancestrales que por cientos de años han mantenido un equilibrio entre la naturaleza y el hombre. Debido a la nueva conciencia ambiental, surgida en las últimas décadas, logrado a través de numerosos convenios y protocolos, se ha comprendido, que los recursos naturales son finitos y que para ser sustentables se requiere implementar políticas ambientales, mejores prácticas de producción y una educación ambiental, coherente con los retos ambientales.
La globalización y la masificación de las nuevas tecnologías de la comunicación e información han llevado un mensaje contundente sobre la situación actual del planeta. Los medios de comunicación masificaron frases como: "cambio climático", "calentamiento global", "especies en vía de extinción", "gases efecto invernadero", "calidad de vida", "conciencia ambiental", "contaminantes antropogénicos", "desarrollo sustentable" entre otros, los cuales han traspasado en las comunidades y en la academia, lo que ha impulsado publicaciones científicas y denuncias públicas sobre el deterioro ambiental. Esto se ha evidenciado en el Seminario de Niza sobre "Interdisciplinariedad en las Universidades", celebrado en septiembre de 1970, que dio lugar al libro Interdisciplinariedad. Problemas de la Enseñanza y de la Investigación en las Universidades.

Los Problemas del Conocimiento y la Perspectiva Ambiental del Desarrollo, han dado inicio a un pensamiento ambiental que habría de ir anidando en diversas universidades de la región como lo son: La Universidad Industrial de Santander (UIS), Universidad Autónoma de Occidente (UAO), Universidad Autónoma del Caribe, Universidad Católica de Colombia, Universidad Central, Universidad Colegio Mayor de Cundinamarca, Universidad Cooperativa de Colombia (UCC), Universidad de Antioquia, Universidad de Cartagena, Universidad de Ciencias Aplicadas y Ambientales (UDCA) Universidad de la Amazonía, Universidad de la Paz, Universidad de la Sabana, Universidad de la Salle, Universidad de los Llanos (UNILLANOS), Universidad de Medellín, Universidad del Cauca, Universidad del Norte, Universidad del Sinú, Universidad Distrital, Universidad El Bosque, Universidad Francisco de Paula Santander, Universidad INCCA De Colombia, Universidad Libre, Universidad Nacional de Colombia- Bogotá - IDEA, Universidad Pedagógica Nacional, Universidad Piloto de Colombia, Universidad Santo Tomás, Universidad Tecnológica de Bolívar, Universidad Tecnológica de Pereira y Universidad Tecnológica del Chocó. A este libro habría de seguir otro, publicado en 1994, sobre Ciencias Sociales y Formación Ambiental, 
enfocado a ambientar las ciencias sociales. A finales de octubre de 1985 se llevó en Bogotá el Primer Seminario sobre Universidad y Medio Ambiente en América Latina y el Caribe, organizado por la Red de Formación Ambiental para América Latina y el Caribe del PNUMA, en colaboración con UNESCO y en el marco del PIEA.

Este escenario, que muestra una realidad de los impactos ambientales y de las consecuencias catastróficas por su alteración, lleva a la creación de programas ambientales en las universidades latinoamericanas. Particularmente para la educación superior, en 1985 se realizó el seminario de Bogotá "Universidad y Medio Ambiente en América Latina y el Caribe", donde se analizó el importante papel que desempeñan las universidades en los procesos de desarrollo y, por lo mismo, el imperativo de vincular la educación superior con la temática ambiental. La cátedra ambiental es común a diversos programas o carreras. La universidad tiene una responsabilidad y la obligación social de fomentar el conocimiento ambiental que es transversal a diferentes programas académicos de diversas áreas del conocimiento como: a) Agronomía, Veterinaria y afines, b) Bellas Artes, c) Ciencias de la Educación, d) Ciencias de la Salud, e) Ciencias Sociales y Humanas, f) Economía, Administración, Contaduría y afines, g) Ingeniería, Arquitectura, Urbanismo y afines, y h) Matemáticas y Ciencias Naturales.

El conocimiento ambiental no es propio de los biólogos, ecologistas o ambientalistas, se requiere el establecimiento de grupos multidisciplinarios trabajando en diversos puntos de la tierra, hablando un lenguaje universal, capaces de abordar una problemática ambiental que no puede entenderse como un suceso acaecido a una región, cuando la realidad muestra un rango global, que sin importar en que territorio ocurra un evento, terminará afectando todo el planeta tierra.

Latinoamérica y el Caribe, no está exenta de las problemáticas ambientales, cambio climático y cambios globales. Tiene selvas tropicales consideradas los pulmones del mundo, los países de la región, disponen de la mayor cantidad de agua dulce y de biodiversidad del planeta. Es una región extensa que parte de la frontera norte de México y se extiende al sur en tierra del Fuego Argentina. Conformada por 41 estados. Se identifican por una historia común y una diversidad cultural.

Los retos ambientales asumidos conllevan responsabilidad de los gobiernos, de la comunidades y de la academia, visibilizado la fragilidad de los ecosistemas por la sobre explotación de los recursos naturales. En 1992, (3 al 14 de junio) se realizó en Rio de Janeiro, Brasil, la Cumbre de la tierra, Conferencia de las Naciones Unidas sobre el Medio Ambiente y el Desarrollo, la cual con 108 Jefes de Estado y de Gobierno y la participación de 172 países.

Por primera vez se establecieron principios que guían el camino de los gobiernos, las comunidades y la academia sobre las responsabilidades ambientales, destacándose:

« Los seres humanos constituyen el centro de las preocupaciones relacionadas con el desarrollo sostenible. Tienen derecho a una vida saludable y productiva en armonía con la naturaleza. ", (Principio 1) «Para alcanzar el desarrollo sostenible, la protección del medio ambiente debe ser parte del proceso de desarrollo y no puede ser considerado por separado ». (Principio 4).

Estos principios transformados en herramientas que marcan una perspectiva sobre acciones a emprender teniendo como postulado el desarrollo sostenible que implica una responsabilidad ambiental, social y económica.

La conferencia de Rio de Janeiro, dejó una serie de documentos y compromisos mundiales sobre la responsabilidad ambiental. Tales documentos son guías a adoptar dentro de las políticas ambientales mundiales, además de la responsabilidad de 
establecer acciones permanentes y dinámicas que busquen mantener un equilibrio ambiental. Estos documentos son dinámicos y se adaptan a las circunstancias ambientales que surjan, de ahí parte la importancia de la socialización y conocimiento.

- Declaración de Río de Janeiro sobre Medio Ambiente y Desarrollo.

- Agenda 21.

- Convención sobre la diversidad biológica.

- Declaración sobre los bosques y masas forestales.

- Convención Marco sobre el Cambio Climático.

La próxima Cumbre de la Tierra Río+20, llamada oficialmente Conferencia de Naciones Unidas sobre Desarrollo Sustentable- se celebró del 20 al 22 de junio de 2012 en Río de Janeiro, Brasil. En este evento se midieron los avances sobre los compromisos pactados 20 años atrás.

La conferencia de Río marco para las universidades de la región, la misión y visión sobre la responsabilidad social ambiental. Las universidades latinoamericanas, han creado redes para tratar problemáticas ambientales. Una de las herramientas de difusión es la organización de simposios, seminarios y conferencias que muestran avances significativos en investigaciones. En noviembre de 1985, se realizó Primer Seminario sobre Universidad y Medio Ambiente en América Latina y el Caribe, realizado en Bogotá. El rol activo y de liderazgo en las comunidades y de las universidades, los gobiernos de la región ha llevado a establecer múltiples actividades de multidisciplinarias.

La Universidad tiene un papel activo y crítico frente a la problemática actual, es consciente desde su autonomía de esta responsabilidad y asume retos en relación con la gestión ambiental y la planificación estratégica que vincula ámbitos de acción social, económica, política y ambiental. Antes de la conferencia de Río de 1992, las universidades habían dado los primeros pasos por una senda ambiental de la cual no puede apartarse por ser parte de la misma esencia que forma toda la naturaleza que estudia. Las universidades fueron las primeras instituciones educativas que en Colombia que respondieron a los retos de la educación ambiental. Desde los primeros años de la década del ochenta, reconocidas instituciones de educación superior comenzaron a ofrecer programas de formación ambiental en posgrado, particularmente maestrías y especializaciones, y a plantearse el problema de la ambientalización de los currículos.

El Programa Internacional de Educación Ambiental (PIEA) creado por UNESCO y el PNUMA en 1975, y la Conferencia Intergubernamental de Tbilisi en 1977, establecieron como prioridad introducir la dimensión ambiental en las universidades. En los años 80 publicaron temáticas sobre la educación ambiental y las ciencias ambientales.

Universidades e investigadores plantaron postulados y reflexión sobre el abordaje de las ciencias ambientales. En México 1976, con la realización del Primer Simposio sobre Ecodesarrollo, de la Asociación Mexicana de Epistemología, de las ciencias ambientales. En noviembre del mismo año el Centro Internacional de Formación en Ciencias Ambientales (CIFCA), establecido por una cooperación entre el gobierno de España y el PNUMA se promovió la reflexión sobre la incorporación de la dimensión ambiental en diversas áreas del conocimiento.

Iniciando los años 80, se forma la Red de Formación Ambiental en América Latina y el Caribe, el CIFCA Iniciativa de Copenhague para Centroamérica y México, organizó seminarios sobre temáticas ambientales, en Colombia. Surgió en 1981 un proyecto colectivo sobre "Articulación de las Ciencias y Gestión Ambiental del Desarrollo", publicando en 1986 de Los Problemas del Conocimiento y la Perspectiva Ambiental del Desarrollo, génesis del pensamiento ambiental de diversas universidades. En 1994, se publicó un segundo libro Ciencias Sociales y Formación Ambiental. 
En 1971, en Colombia, se llevó a cabo el Comité "Hombre y biosfera" del "Grupo de Desarrollo Tecnológico, Cultura y Estudios Prospectivos Año 2000", se constituyó en el Comité Nacional del Programa MAB (Man and Biosphere) en Colombia y en octubre de 1985, en Bogotá, se realizó el Primer Seminario sobre Universidad y Medio Ambiente en América Latina y el Caribe, organizado, por la Red del PNUMA, "Formación Ambiental para América Latina y el Caribe", en colaboración con UNESCO y PIEA. Participaron 59 universidades de 22 países y se analizó la internalización de la dimensión ambiental en las disciplinas de las ciencias naturales, sociales, tecnológicas y de la salud: Publicándose una Carta de Bogotá sobre Universidad y Medio Ambiente y 10 Tesis sobre Medio Ambiente.

De esta manera, se inició una discusión continua y disciplinada, luego se realizaron entre 1987 y 1990, 3 seminarios de Universidad y Medio Ambiente en Argentina y 4 seminarios en Brasil. Colombia celebró 3 seminarios (1999 en Cali; 2005 en Bogotá y 2007 en Bogotá). 5 Congresos Iberoamericanos de Educación Ambiental celebrados en México, Venezuela, Cuba y Brasil entre 1992 y 2006 han dado un estímulo y han marcado el camino a las universidades latinoamericanas, estableciendo redes y formando facultades con programas ambientales de pregrado y posgrado.

Toda esta revolución del pensamiento ambiental planteó distintos enfoques epistemológicos del área del conocimiento ambiental surgiendo corrientes de "pensamiento ambiental", "saber ambiental", "estudios ambientales" y "ciencias ambientales", basadas en diferentes puntos de vista filosóficos, éticos, políticos y epistemológicos.

\section{Conclusiones}

La temática ambiental involucra a las universidades en todas las áreas del conocimiento. Estas temáticas se han construido lentamente durante los últimos 25 años. El reto de la crisis ambiental surgida durante los años 70, conllevó a establecer estrategias que mitigaran la enorme complejidad de la crisis ambiental. Resultado del excesivo usufructo de los recursos naturales, contaminación de ríos y mares, desaparición de especies, cambio climático y agotamiento de los recursos. En 1980, se estableció la relación primordial que hay entre las actividades económicas y el medio ambiente, en 1987 en el informe de Brundtland de la comisión mundial de medio ambiente y desarrollo (CM$\mathrm{MAD}$ ), proclamó el desarrollo sostenible como objetivo central de la economía. El desarrollo sostenible entendido como "Aquel que es capaz de cubrir las necesidades del presente sin comprometer las posibilidades de las futuras generaciones para satisfacer sus propias necesidades" (CMMAD, 1992).

Todos estos paradigmas traen un gran reto a las universidades latinoamericanas, las cuales desde la percepción de la realidad y su compromiso social ambiental, proponen y posibilitan el desarrollo de las comunidades. La universidad es autónoma en los caminos que decide recorrer; por lo tanto, es reflexiva y ética sobre el actuar de las realidades ambientales que afectan las regiones.

La academia en Latinoamérica es pilar fundamental para forjar una cultura que nos permita generar un forma de vida sostenible, buscando siempre el balance entre los diferentes aspectos que influyen en el desarrollo humano. 


\section{Literatura citada}

1. ACIUR. (1999). "Lineamientos para una Política Nacional de Investigación en Temas de Hábitat:'Asociación Colombiana de Investigadores Urbano Regionales. En: Ciencias del Medio Ambiente y del Hábitat. Plan Estratégico 19992004. Instituto Colombiano para el Desarrollo de la Ciencia y la Tecnología Bogotá: "Francisco José de Caldas".

2. Amaya, P. (1988). Ciencia, tecnología y medio ambiente. En: Memorias del Seminario Internacional Ecobios Colombia 88. El desarrollo sostenible: estrategias, políticas y acciones. Bogotá: Instituto Nacional de los Recursos Naturales -Inderena.

3. Amaya, P. (1989). Colombia. Importancia de la investigación en la gestión ambiental. En: Colombia. Gestión ambiental para el desarrollo. Bogotá: Instituto Nacional de los Recursos Naturales -Inderena- y Sociedad Colombiana de Ecología.

4. Ángel, A. (1988). Estudio sobre el estado de desarrollo e inserción social de la ecología y las ciencias ambientales. Síntesis. Fondo Colombiano de Investigaciones Científicas y Proyectos Especiales "Francisco José de Caldas.' Bogotá.

5. Becher, T. (2001). Tribus y territorios académicos. La indagación intelectual y las culturas de las disciplinas. Barcelona, Gedisa Editorial.

6. Carson, R. Silent Spring (Boston: Houghton Mifflin, 1962), Mariner Books, 2002, ISBN 0-618-24906-0.

7. Colciencias. (1971). Programa Nacional de Ecología y Ciencias Ambientales. Fondo Colombiano de Investigaciones Científicas y Proyectos Especiales. Bogotá: "Francisco José de Caldas".
8. Colciencias. (1973). Facultades de Ingeniería. Retos en la formación del ingeniero para el año 2020 - Acofi. Cartagena de Indias, Colombia. 20 al 22 de septiembre.

9. García, G. (2007). Surgimiento y evolución de la Ingeniería Ambiental en Colombia. Bogotá. Disponible en http://www.riourbano.org/images/documentos/surgimientoevolucion ing ambiental.pdf,

10. Gómez, L. (2002) Introducción a la ecología global. Facultad de Ciencias Humanas y Económicas. Medellín, Universidad Nacional,

11. Grupo de Desarrollo Tecnológico y Prospectiva. Grupo del año 2000. Fondo Colombiano de Investigaciones Científicas y Proyectos Especiales "Francisco José de Caldas".

12. Leff, E. (2005). Complejidad, racionalidad ambiental y diálogo de saberes. Buenos Aires (Argentina). Seminario Iberoamericano de Formación Ambiental. 21-22 de septiembre, 2005.

13. Morin, E. (2001). El Método IV. Las ideas. Su hábitat, su vida, sus costumbres, su organización. Madrid, Cátedra.

14. Morin, E. (2003). El Método V. La humanidad de la humanidad. La identidad humana. Madrid, Cátedra. Prospectiva No. 4. Bogotá.

15. Toulmin, S. (1977). La comprensión humana. (Vol. I). "El uso colectivo y la evolución de los conceptos". Madrid, Alianza Editorial. 